\title{
The Expression, Morphology, and Clinical Characteristics of Fibroblast Growth Factor-10 in Breast Cancer
}

\author{
Samira Molashahi', Dariush D Farhud ${ }^{2}$, Mojtaba Saffari ${ }^{3}$, Elmira Ebrahimi', \\ Amirnader Emamirazavi ${ }^{4}$, Reza Shirkoohi ${ }^{1}$
}

${ }^{1}$ Cancer research center, Cancer Institute of Iran, Tehran University of Medical Sciences, Tehran, Iran. ${ }^{2}$ School of Public Health, Tehran University of Medical Sciences, Tehran, Iran. ${ }^{3}$ Department of Genetics, Faculty of Medicine, Tehran University of Medical Sciences, Tehran, Iran. ${ }^{4}$ Iran National Tumor Bank, Cancer Institute of Iran, Tehran University of Medical Sciences, Tehran, Iran.

\begin{abstract}
Background: Fibroblast growth factor-10 (FGF-10) is a member of a superfamily with characteristics of epithelial cell proliferation and embryonic development and is assumed to have a role in a phenomenon called the epithelial-mesenchymal transition (EMT). The previous study has revealed the critical role of FGF-10 in type III EMT in breast cancer cell lines. The mentioned finding, demonstrates the possible role of this factor in type III EMT in cancers with different origins such as breast. The present study investigated the expression of FGF-10 as a mitotic-inducing growth factor, normally has a low expression in breast tissues amongst breast cancer patients. Materials and Methods: 67 breast cancer tissues and 8 normal breast tissues were randomly selected from the Iran national tumor bank. The FGF-10 gene expression analysis was performed after the RNA expression using the real-time RTPCR, which was followed by the Student's t-test statistical analysis. Results: The findings revealed that the relative expression of FGF-10 was elevated in tumor tissues as compared with normal breast tissues, and the higher expression had a direct correlation with the progression of clinical and pathologic staging. The expression was also significantly higher in triple-negative breast cancer and p53 null tissues. Conclusion: Taken together, it is suggested that, although in some variables it was not significant but generally the invasion and migration in tumor tissues are the same as in-vitro analysis as indicated before, and the expression has a direct relationship with the molecular presentation and clinical -pathological progression.
\end{abstract}

Keywords: Breast Cancer- FGF-10- triple negative breast cancer- P53

Asian Pac J Cancer Biol, 6 (3), 219-224

\section{Introduction}

Fibroblast Growth Factor (FGF), a member of a growth-inducer family, is a heparin-binding protein and plays a role in various phenomena including proliferation, development, angiogenesis, and embryonic development [1]. FGF has almost 22 members [2]. The related receptors in mammalian cells are identified as FGFR1-4 [3]. FGF-10 is a member of this family with characteristics of epithelial cell proliferation and embryonic development and is supposed to have a role in a phenomenon called the epithelial-mesenchymal transition (EMT) [4].

EMT is a harmonic orchestra that can induce mesenchymal markers and features [5]. Embryonic development is associated with type I EMT, which
Submission Date: 09/08/2021_Acceptance Date: 10/01/2021

\footnotetext{
Corresponding Author:

Dr. Reza Shirkoohi

Cancer Biology Research Center, Cancer Institute of Iran, Keshavarz Blvd, Imam Khomeini Hospital Complex, Tehran, Iran.

Email: rshirkoohi@tums.ac.ir
}

leads to organogenesis [6]. Several functions have been considered for FGF-10 in the early stage of the embryonic development $[7,8]$. In contrast, type III EMT is accompanied by a bizarre disturbance in differentiation pathways and is finally associated with the invasion and metastasis [5]. The present researchers' previous study has revealed the critical role of FGF-10 in type III EMT in breast cancer cell lines. The mentioned finding demonstrates the possible role of this factor in type III EMT in cancers such as breast with different origins [4].

Breast cancer is the most common cause of cancer deaths among women and has an increasing incidence rate in Iran [9]. Although recent technologies developed 
for screening this cancer have increased the survival rate of breast cancer, there is yet a long way ahead to manage and control the outcome of patients suffering from breast cancer [10]. Specification of the factors affecting the prognosis would be very critical to achieve the mentioned goal.

Cell morphology findings and clinical staging are two different indicators that can be employed for the prognostic estimation and clinical management of breast cancer [11]. The comparisons made between these indicators and gene profile would provide a better insight for obtaining a more precise prediction in this regard [11]. In recent years, the molecular classification has helped to identify the prognosis and outcome of breast cancer more precisely by the administration of drugs that can directly target critical molecules in different signaling pathways. In this case, the classification of molecular subtypes according to the hormone receptor expression (luminal A, luminal B, Her2-enriched, and basal-like) has become an initial routine management of breast cancer [12]. The present study has investigated the expression of FGF-10 as a mitotic-inducing growth factor, which normally has a low expression in breast tissues amongst breast cancer patients.

\section{Materials and Methods}

\section{Patients and tumor preparation}

The present cross-sectional study involved 75 patients that were randomly selected from Iran national tumor bank (Cancer institute of Iran, Tehran University of Medical Sciences, Tehran, Iran). The mentioned sample included 67 breast tumor tissues as well as 8 breast tissues from patients that referred for mammoplasty without a breast cancer history. The clinical morphologic and immunohistochemical (IHC) information was received from tumor bank data base ( including lymph node and perineural invasion, metastasis, grade, stage, estrogen, progestron, Her2 receptor and p53 expression). All fresh tissues were stored at -80 for further procedures. An informed consent was obtained from all the patients.

Demographic, morphologic, and clinical information of patients were entered into the excel file (Microsoft 2010, USA).

\section{Gene primer design}

Primers were designed using primer3 that is an online primer designer (http://bioinfo.ut.ee/ primer3-0.4.0/primer3/). Primer sequences were 5'-ATGTCCGCTGGAGAAAGCTA-3' and 5'-CCCCTTCTTGTTCATGGCTA-3' as forward and reverse primers, respectively. GAPDH was also designed as a housekeeping gene following the same method with 5'-TCACCAGGGCTGCTTTTAAC-3' and 5'-GACAAGCTTCCCGTTCTCAG-3' sequences as forward and reverse primers, respectively. Primer-blast online tool was used to confirm the specific product and predict to avoid non- specific primer-annealing products (https://www.ncbi.nlm.nih.gov/tools/primer-blast).

\section{RNA extraction}

RNA was extracted using easy-BLUE (iNtRON, South Korea) according to the manufacturer's instruction. The quantity of the extracted RNA was analyzed using the NanoDrop spectrophotometer (Thermo, USA).

\section{Real time RT-PCR}

The cDNA was constructed based on the construction manual of cDNA synthesis kit (Takara, Japan). The 1ug of the extracted RNA was used for cDNA synthesis. The cDNA synthesis was checked by PCR for GAPDH gene, and the following procedures were performed. 2 ul of cDNA was transferred to SYBER Green Master Mix to perform the real-time PCR (Takara, Japan). Real-time PCR was performed using the Rotor-Gene Q thermocycler (Qiagen, USA), which has been described previously [4].

\section{Data transfer and statistics}

Data for the gene amplification was analyzed by Rotor-Gene Q software (Qiagen, USA). The fold change of expression was analyzed using REST software (Qiagen, USA) to perform det and ddct calculations. To compare tumor and normal tissue gene expression det of tumor and normal tissue was analyzed. In case of clinical and morphologic correlation, data of ddct was used. The data of the normal tissues was pooled, and the average value was calculated. The association was also addressed by calculating the p-value and employing the Student's t-test

\section{Results}

\section{Demographic analysis}

A total number of 67 breast cancer tumor tissues were studied for the expression of FGF-10. The mean age of patients was 47.52 with the maximum and minimum range of 74 and 31 years old, respectively. The pathologic distribution presented as the number (percentage) was 8 (11.98\%), 33 (49.25\%), 21 (31.34\%), and 5 (7.47\%) for grades I, II, III, and X (unknown), respectively. Moreover, the pathologic distribution presented as the number (percentage) was $2(2.99 \%), 39(58.22 \%), 25$ (37.31\%), and $1(1.48 \%)$ for clinical stages I, II, III, and IV, respectively.

\section{Expression of FGF-10 in tumor tissues}

Dct comparison made between tumor tissues and pooled normal tissues revealed the higher expression of FGF-10 in tumor tissues ( $p=0.00008$ ). The expression comparison is illustrated in Figure. 1. a.

\section{FGF-10 expression and clinical/morphological findings}

The relative expression of FGF-10 was found to increase by decreasing the differentiation of tumor. Moreover, the expression was higher in grade II as compared with grades I and III; however, it was not significant among different grades (Figure. 1. b). The same pattern was observed concerning the stages of the disease, and the expression of FGF-10 was higher in stage II as compared with the other clinical stages (Figure.1. c). 


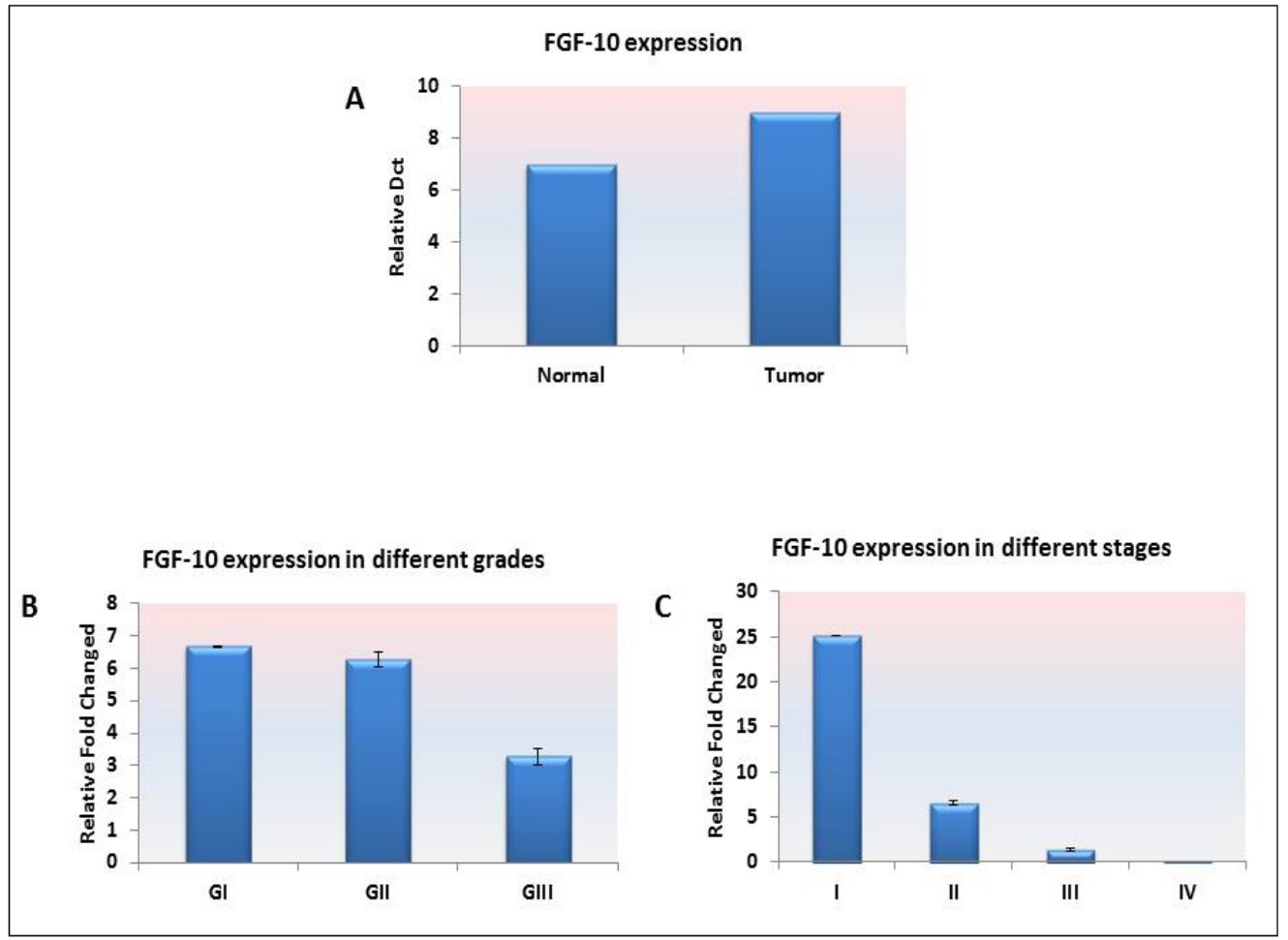

Figure 1. A. The Average of the FGF-10 Expression was Significantly (*) Higher in Tumor Tissues as Compared with Pooled Normal Breast Tissues $(p=0.00008)$. B. The expression of FGF-10 was increasing with the progression of the pathologic grade and was higher in grade II; however, there was no statistical significance in this regard. C. The same pattern reported for the grade was observed for the clinical stage and was also more evident in stage II; however, there was no statistical significance among stages.

\section{FGF-10 expression and invasion}

The relative expression of FGF-10 was found to be higher in tumors with perineural (Figure. 2. a) and vascular (Figure. 2. b) invasion; however, it was not significant.

\section{FGF-10 expression and molecular pattern}

Number of 19 samples was triple negative based on immunohistochemistry report. Also 13 samples were expressing HER2 receptor expression. The findings have shown that the relative expression of FGF-10 was significantly lower in triple-negative tumor tissues as compared with tumors with at least one receptor expression (Figure. 3. a) $(\mathrm{p}=0.04)$. However, there was no significant difference concerning the expression of the HER-2 receptor (Figure. 3.b). In contrast, the expression of FGF-10 was significantly higher in tumor tissues with no expression of p53 (Figure. 3. c) $(\mathrm{p}=0.03)$.

\section{Discussion}

The present researchers have previously demonstrated that the FGF-10 up-regulation has a positive effect on the invasion and migration in breast cancer cell lines [4]. The

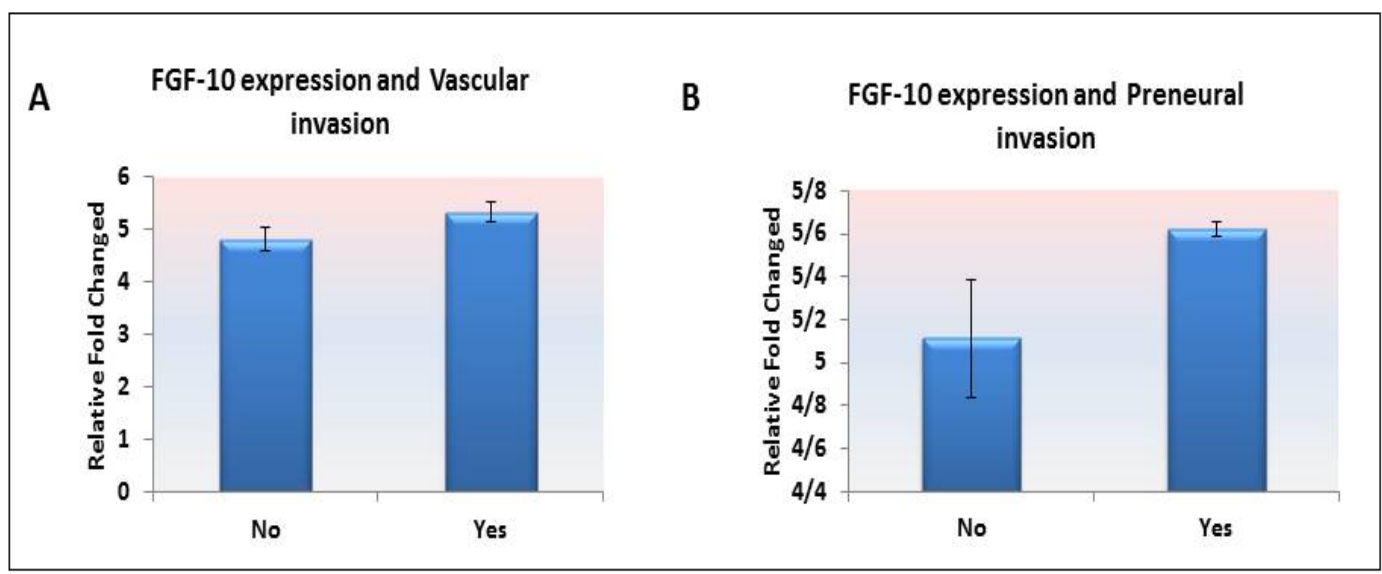

Figure 2. The Relative Expression of FGF-10 was Higher among Tissues with (A) Vascular Invasion and (B) Perineural Invasion; However, This Association was not Significant. 


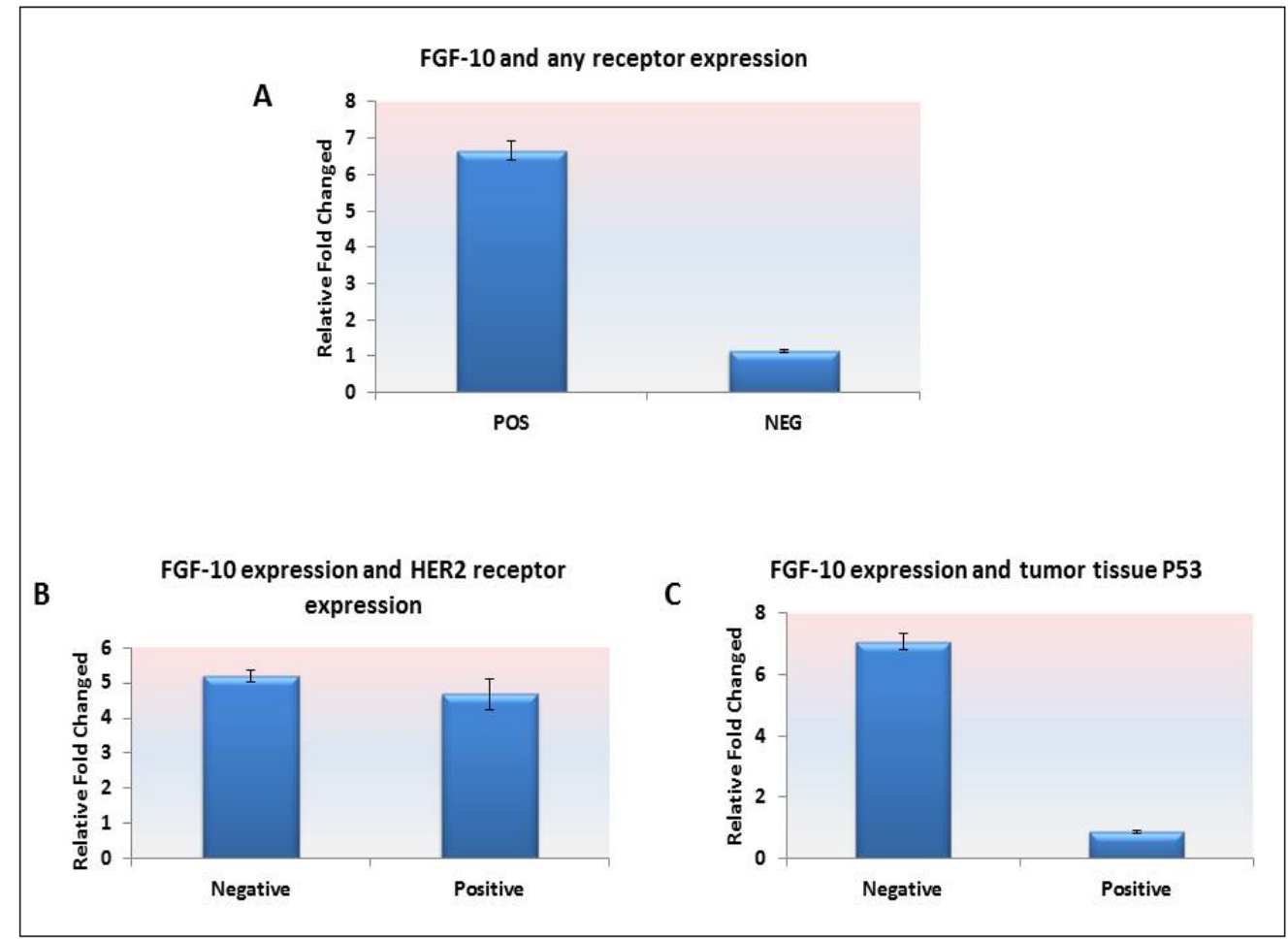

Figure 3. A. The Relative Expression of FGF-10 was Significantly (*) Higher among Tissues with Triple-negative Receptor $(\mathrm{p}=0.04)$. B. Comparison made between HER2- positive and -negative tumor tissues did not show a significant difference in the relative gene expression of FGF-10. C. The relative expression of FGF-10 was higher among tumors without P53 expression $(*)(\mathrm{p}=0.03)$.

higher expression of FGF-10 means that the expression of this critical protein in type I EMT may have a role in a considerable number of breast cancer tumor tissues [4]. In type I EMT, the expression of FGF-10 during the gastrulation and kidney development is very critical [13]. Meanwhile, the present researchers' previous findings have revealed that the regulation of this protein can change the cancer cell behavior [4]. The EMT is a harmonic orchestration, which is a critical process in the early stage of life $[5,14]$. In this phenomenon, uniform and regular epithelial cells morphologically and functionally change to the spindle-shaped mesenchymal cells with the ability of movement and migration [14]. During the first stages of life, this phenomenon is a serious process for the development and organogenesis that is recognized as type I [6]. Although type II is also important during the inflammation, type III is known to happen during metastasis [6]. In this case, there are several studies showing that embryonic factors also have a role in the cancer progression. The mentioned finding makes the possible role of FGF-10 in breast cancer more prominent [5]

The present study revealed that the expression of FGF-10 was higher in breast cancer tumor tissues. The expression of FGF-10 was higher in grade and stage II as compared with other clinical and morphological characteristics. As we have previously indicated in colorectal carcinoma cell lines and tumor tissues, the expression of FGF-10 increased by increasing the grades and stages (specially stage III) [15].

There are several recognized signaling pathways for
FGF-10 including Ras/MAPK, AKT/mTOR, TGFb, and wnt signaling pathways through the phosphorylation of GSK3b [16-19]. The invasion indexes in tumor tissues have indicated a slight increase in the expression of FGF10 in more invasive tumor cells. The mentioned finding has been confirmed with respect to breast cancer cell lines, as well. Concerning the correlation with FGF-10 expression and invasion in tumor tissues, one reason for insignificant results in comparison with the cell line might be because of the tumor heterogeneity [20]. There are different variables including the percentage of normal tissues or lymphocytic infiltration, the duration of tumor tissue preparation after excision, and environmental factors such as hypoxia that can affect the results of the relative gene expression. In such cases, the gene expression in tumor excision might be different from that of the community of unique cells that are derived from a cloned stable cell line [21].

The classification of breast cancer types based on immune-phenotyping (ER or Estrogen Receptor, PR or Progesterone Receptor, HER2 or Human Epidermal Growth Factor Receptor 2) helps to the better management of the disease and presentation of a breast cancer targeted therapy $[12,22]$. In this case, the tumor tissues that have no immune-phenotypic feature are called triple-negative or basal-like breast cancer. The findings of the present study have shown that the expression of FGF-10 is higher in tumors with at least one receptor-positive as compared with a triple-negative. Triple-negative breast cancer that is the most aggressive type accounts for approximately $20 \%$ of breast cancer subtypes [23]. 
The FGF/FGFR system can be activated aberrantly in a ligand-dependent or -independent manner in breast cancer as various kinds of cancers and has a different role in invasion and drug resistance. Considering that FGF-10 usually binds to FGFR2, it has been revealed that the amplification and overexpression of FGFR2 could be detectable in only $4 \%$ of TNBC cases, which might explain the downregulation of FGF-10 expression in our study [24].

On the other hand, several studies have indicated the correlation between FGFR overexpression and hormone receptors in the cancer development and proliferation. Studies have shown the correlation between the expressions of different hormone receptors and the expression of other family members of FGF superfamily. For example, with respect to the HER2 receptor, there was a direct correlation between FGF-14 and Her2 receptor and the invasion in breast cancer [25]. Moreover, it has been already revealed that the FGF/FGFR inhibition could promote the endocrine therapy [26]. The findings of the present study did not demonstrate any significant difference among hormone receptor positive tumors and the expression of FGF-10.

The expression of FGF-10 was lower in tumor tissues without the expression of $\mathrm{p} 53$. Studies have shown that almost $80 \%$ of TNBC cases have the loss of $\mathrm{p} 53$, which correlates with the poor prognosis. In our study, 76\% of TNBC cases have lost the expression of p53. Some studies have shown that $\mathrm{p} 53$ tumor suppressor has a role in the inhibition of other members of FGF family such as FGF-13 [27]. It can be conjectured that other members such as FGF-10 that has a different signaling pathway to the cell proliferation and invasion might have a possible correlation with the loss of p53 [28].

In conclusion, taken together, it is suggested that the invasion and migration in tumor tissues are the same as in-vitro analysis as indicated in the present researchers' previous study, and the expression has a direct relationship with the molecular presentation and clinical-pathological progression. Investigations with sufficient tissue samples might pave the way to shed light on the mentioned difference statistically.

\section{Acknowledgements}

The study was supported by Cancer Research Center, Cancer Institute of Iran, Tehran University of Medical Sciences, Tehran, Iran.

\section{References}

1. Tanner Y, Grose RP. Dysregulated FGF signalling in neoplastic disorders. Seminars in Cell \& Developmental Biology. 2016 05;53:126-135. https://doi.org/10.1016/j. semcdb.2015.10.012

2. Teishima J, Hayashi T, Nagamatsu H, Shoji K, Shikuma H, Yamanaka R, Sekino Y, Goto K, Inoue S, Matsubara A. Fibroblast Growth Factor Family in the Progression of Prostate Cancer. Journal of Clinical Medicine. 201902 04;8(2):183. https://doi.org/10.3390/jcm8020183
3. Yun Y, Won JE, Jeon E, Lee S, Kang W, Jo H, Jang J, Shin US, Kim H. Fibroblast Growth Factors: Biology, Function, and Application for Tissue Regeneration. Day R. Journal of Tissue Engineering. 2010 01;1(1):218142. https://doi. org/10.4061/2010/218142

4. Abolhassani A, Riazi GH, Azizi E, Amanpour S, Muhammadnejad S, Haddadi M, Zekri A, Shirkoohi R. FGF10: Type III Epithelial Mesenchymal Transition and Invasion in Breast Cancer Cell Lines. Journal of Cancer. 2014;5(7):537-547. https://doi.org/10.7150/jca.7797

5. Shirkoohi R. Epithelial mesenchymal transition from a natural gestational orchestration to a bizarre cancer disturbance. Cancer Science. 2012 Dec 31;104(1):28-35. https://doi. org/10.1111/cas. 12074

6. Nakaya Y, Sheng G. EMT in developmental morphogenesis. Cancer Letters. 2013 Nov;341(1):9-15. https://doi. org/10.1016/j.canlet.2013.02.037

7. Chan SS, Li H, Hsueh Y, Lee DS, Chen J, Hwang S, Chen C, Shih E, Hsieh PCH. Fibroblast Growth Factor-10 Promotes Cardiomyocyte Differentiation from Embryonic and Induced Pluripotent Stem Cells. Whitsett JA. PLoS ONE. 2010 Dec 28;5(12):e14414. https://doi.org/10.1371/journal. pone. 0014414

8. Koleganova N, Piecha G, Ritz E, Becker LE, Müller A, Weckbach M, Nyengaard JR, Schirmacher P, GrossWeissmann M. Both high and low maternal salt intake in pregnancy alter kidney development in the offspring. American Journal of Physiology-Renal Physiology. 2011 08;301(2):F344-F354. https://doi.org/10.1152/ ajprenal.00626.2010

9. Enayatrad M, Amoori N, Salehiniya H. Epidemiology and trends in breast cancer mortality in iran. Iran J Public Health. 2015;44(3):430-1.

10. Tinoco G, Warsch S, Glück S, Avancha K, Montero AJ. Treating Breast Cancer in the 21st Century: Emerging Biological Therapies. Journal of Cancer. 2013;4(2):117-132. https://doi.org/10.7150/jca.4925

11. Xie Y, Su N, Yang J, Tan Q, Huang S, Jin M, Ni Z, Zhang B, Zhang D, Luo F, Chen H, Sun X, Feng JQ, Qi H, Chen L. FGF/FGFR signaling in health and disease. Signal Transduction and Targeted Therapy. 202009 02;5(1). https:// doi.org/10.1038/s41392-020-00222-7

12. Tsang JY, Tse GM. Molecular Classification of Breast Cancer. Advances in Anatomic Pathology. 201904 30;27(1):27-35. https://doi.org/10.1097/pap.0000000000000232

13. Michos O, Cebrian C, Hyink D, Grieshammer U, Williams L, D'Agati V, Licht JD, Martin GR, Costantini F. Kidney Development in the Absence of Gdnf and Spry1 Requires Fgf10. Beier DR. PLoS Genetics. 201001 15;6(1):e1000809. https://doi.org/10.1371/journal.pgen.1000809

14. Aiello NM, Stanger BZ. Echoes of the embryo: using the developmental biology toolkit to study cancer. Disease Models \& Mechanisms. 201602 01;9(2):105-114. https:// doi.org/10.1242/dmm.023184

15. Farajihaye Qazvini F, Samadi N, Saffari M, Emami-Razavi AN, Shirkoohi R. Fibroblast growth factor-10 and epithelialmesenchymal transition in colorectal cancer. EXCLI Journal; 18:Doc530; ISSN 1611-2156. 2019; . https://doi. org/10.17179/EXCLI2018-1784

16. Masola V, Gambaro G, Tibaldi E, Brunati AM, Gastaldello A, D'Angelo A, Onisto M, Lupo A. Heparanase and Syndecan-1 Interplay Orchestrates Fibroblast Growth Factor-2-induced Epithelial-Mesenchymal Transition in Renal Tubular Cells. Journal of Biological Chemistry. 2012 01;287(2):1478-1488. https://doi.org/10.1074/jbc.m111.279836

17. Hardy KM, Yatskievych TA, Konieczka J, Bobbs AS, Antin 
PB. FGF signalling through RAS/MAPK and PI3K pathways regulates cell movement and gene expression in the chicken primitive streak without affecting E-cadherin expression. BMC Developmental Biology. 201103 21;11(1). https:// doi.org/10.1186/1471-213x-11-20

18. Buchtova M, Oralova V, Aklian A, Masek J, Vesela I, Ouyang Z, Obadalova T, Konecna Z, Spoustova T, Pospisilova T, Matula P, Varecha M, Balek L, Gudernova I, Jelinkova I, Duran I, Cervenkova I, Murakami S, Kozubik A, Dvorak P, Bryja V, Krejci P. Fibroblast growth factor and canonical WNT/ $\beta$-catenin signaling cooperate in suppression of chondrocyte differentiation in experimental models of FGFR signaling in cartilage. Biochimica et Biophysica Acta (BBA) - Molecular Basis of Disease. 2015 05;1852(5):839-850. https://doi.org/10.1016/j.bbadis.2014.12.020

19. Ooshima A, Park J, Kim S. Phosphorylation status at Smad3 linker region modulates transforming growth factor- $\beta$ induced epithelial-mesenchymal transition and cancer progression. Cancer Science. 201901 23;110(2):481-488. https://doi.org/10.1111/cas.13922

20. Strickaert A, Saiselet M, Dom G, De Deken X, Dumont JE, Feron O, Sonveaux P, Maenhaut C. Cancer heterogeneity is not compatible with one unique cancer cell metabolic map. Oncogene. 2016 Oct 31;36(19):2637-2642. https:// doi.org/10.1038/onc.2016.411

21. Pearce DA, Arthur LM, Turnbull AK, Renshaw L, Sabine VS, Thomas JS, Bartlett JMS, Dixon JM, Sims AH. Tumour sampling method can significantly influence gene expression profiles derived from neoadjuvant window studies. Scientific Reports. 201607 07;6(1). https://doi.org/10.1038/srep29434

22. Al-thoubaity FK. Molecular classification of breast cancer: A retrospective cohort study. Annals of Medicine and Surgery. 2020 01;49:44-48. https://doi.org/10.1016/j. amsu.2019.11.021

23. Chew NJ, Nguyen EV, Su S, Novy K, Chan HC, Nguyen LK, Luu J, Simpson KJ, Lee RS, Daly RJ. FGFR3 signaling and function in triple negative breast cancer. Cell Communication and Signaling. 202001 27;18(1). https:// doi.org/10.1186/s12964-019-0486-4

24. Santolla MF, Maggiolini M. The FGF/FGFR System in Breast Cancer: Oncogenic Features and Therapeutic Perspectives. Cancers. 2020 Oct 18;12(10):3029. https:// doi.org/10.3390/cancers12103029

25. Willis AL, Tran NL, Chatigny JM, Charlton N, Vu H, Brown SA, Black MA, McDonough WS, Fortin SP, Niska JR, Winkles JA, Cunliffe HE. The Fibroblast Growth FactorInducible 14 Receptor Is Highly Expressed in HER2-Positive Breast Tumors and Regulates Breast Cancer Cell Invasive Capacity. Molecular Cancer Research. 2008 05;6(5):725734. https://doi.org/10.1158/1541-7786.mcr-08-0005

26. Perez-Garcia J, Muñoz-Couselo E, Soberino J, Racca F, Cortes J. Targeting FGFR pathway in breast cancer. The Breast. 2018 02;37:126-133. https://doi.org/10.1016/j. breast.2017.10.014

27. Manfredi JJ. Tumor suppression by p53 involves inhibiting an enabler, FGF13. Proceedings of the National Academy of Sciences. 201701 12;114(4):632-633. https://doi. org/10.1073/pnas.1619815114

28. Li J, Zhang X, Zhang Z, Zheng L, Jindal S, Liu Y. Association of p53 expression with poor prognosis in patients with triple-negative breast invasive ductal carcinoma. Medicine. 2019 05;98(18):e15449. https://doi.org/10.1097/ md.0000000000015449

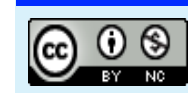

This work is licensed under a Creative Commons AttributionNon Commercial 4.0 International License. 